\title{
Expected geoneutrino signal at JUNO
}

\author{
Virginia Strati ${ }^{1,2^{*}}$, Marica Baldoncini ${ }^{1,3}$, Ivan Callegari ${ }^{2}$, Fabio Mantovani ${ }^{1,3}$, William F McDonough ${ }^{4}$, Barbara Ricci ${ }^{1,3}$ \\ and Gerti Xhixha ${ }^{2}$
}

\begin{abstract}
Constraints on the Earth's composition and on its radiogenic energy budget come from the detection of geoneutrinos. The Kamioka Liquid scintillator Antineutrino Detector (KamLAND) and Borexino experiments recently reported the geoneutrino flux, which reflects the amount and distribution of $U$ and Th inside the Earth. The Jiangmen Underground Neutrino Observatory (JUNO) neutrino experiment, designed as a 20 kton liquid scintillator detector, will be built in an underground laboratory in South China about $53 \mathrm{~km}$ from the Yangjiang and Taishan nuclear power plants, each one having a planned thermal power of approximately $18 \mathrm{GW}$. Given the large detector mass and the intense reactor antineutrino flux, JUNO aims not only to collect high statistics antineutrino signals from reactors but also to address the challenge of discriminating the geoneutrino signal from the reactor background. The predicted geoneutrino signal at JUNO is $39.7_{-5.2}^{+6.5}$ terrestrial neutrino unit (TNU), based on the existing reference Earth model, with the dominant source of uncertainty coming from the modeling of the compositional variability in the local upper crust that surrounds (out to approximately $500 \mathrm{~km}$ ) the detector. A special focus is dedicated to the $6^{\circ} \times 4^{\circ}$ local crust surrounding the detector which is estimated to contribute for the $44 \%$ of the signal. On the basis of a worldwide reference model for reactor antineutrinos, the ratio between reactor antineutrino and geoneutrino signals in the geoneutrino energy window is estimated to be 0.7 considering reactors operating in year 2013 and reaches a value of 8.9 by adding the contribution of the future nuclear power plants. In order to extract useful information about the mantle's composition, a refinement of the abundance and distribution of $U$ and $T h$ in the local crust is required, with particular attention to the geochemical characterization of the accessible upper crust where $47 \%$ of the expected geoneutrino signal originates and this region contributes the major source of uncertainty.
\end{abstract}

Keywords: Geoneutrino flux; JUNO experiment; Earth reference model; Earth composition; Heat-producing elements; Reactor antineutrinos

\section{Background}

The first experimental evidence of geoneutrinos, i.e., electron antineutrinos produced in beta decays along the ${ }^{238} \mathrm{U}$ and ${ }^{232}$ Th decay chains, was claimed by the Kamioka Liquid scintillator Antineutrino Detector (KamLAND) Collaboration in 2005 (KamLAND Collaboration 2005), which ushered in a new method for exploring the Earth's interior and provided constraints on the planet's composition and specifically its radiogenic element budget (Fiorentini et al. 2007). The geoneutrino energy spectrum contains in it distinctive contributions from $\mathrm{U}$ and $\mathrm{Th}$, each one resulting from different rates and

\footnotetext{
*Correspondence: strati@fe.infn.it

'Department of Physics and Earth Sciences, University of Ferrara, Via Saragat 1, 44121 Ferrara, Italy

'Legnaro National Laboratories, INFN, Viale dell'Università, 2, 35020 Legnaro, Italy

Full list of author information is available at the end of the article
}

shapes of their decays (see Figures three and five of Fiorentini et al. 2007) and from concentrations and spatial distributions of these elements inside the Earth.

Geoneutrinos are measured in liquid scintillation detectors via the inverse beta decay (IBD) reaction on free protons:

$$
\bar{v}_{\mathrm{e}}+p \rightarrow e^{+}+n
$$

whose energy threshold of $1.806 \mathrm{MeV}$ means that only a small fraction of the antineutrinos produced from the $\mathrm{U}$ and Th decay chains are detectable. The IBD detection event in a liquid scintillator produces two flashes of light: the annihilation flash, from electron-positron interaction, followed by the deuterium formation flash, which is $2.2 \mathrm{MeV}$ of light that follows some $200 \mu$ s later. The delayed coincidence of these two flashes of light provides the critical identification of the antineutrino

\section{实}


interaction and eliminates most background events. The KamLAND and Borexino experiments recently reported $116_{-27}^{+28}$ geoneutrino events over 2,991 days (KamLAND Collaboration 2013) and $14.3 \pm 4.4$ geoneutrino events in 1,353 days (Borexino Collaboration 2013), respectively. Differences in the detection rates reflect the detector sizes, with the KamLAND detector being approximately $1 \mathrm{kton}$ and the Borexino detector $0.3 \mathrm{kton}$.

The most significant source of background for geoneutrino measurements is due to reactor antineutrinos, i.e., electron antineutrinos emitted during the beta decays of fission products from ${ }^{235} \mathrm{U},{ }^{238} \mathrm{U},{ }^{239} \mathrm{Pu}$, and ${ }^{241} \mathrm{Pu}$ burning. Approximately $30 \%$ of the reactor antineutrino events are recorded in the geoneutrino energy window extending from the IBD threshold up to the endpoint of the ${ }^{214} \mathrm{Bi}$ beta decay spectrum (3.272 MeV) (Fiorentini et al. 2010). The Terrestrial Neutrino Unit (TNU), which is the signal that corresponds to one IBD event per $10^{32}$ free protons per year at $100 \%$ efficiency, is used to compare the different integrated spectral components (i.e., antineutrinos from $\mathrm{U}, \mathrm{Th}$, and reactors) measured by the detectors or just beneath the Earth's surface.

In the past decade, reactor antineutrino experiments played a decisive role in unraveling the neutrino puzzle, which currently recognizes three flavor eigenstates $\left(v_{\mathrm{e}}\right.$, $v_{\mu}$, and $v_{\mathrm{T}}$ ), each of which mixes with three mass eigenstates $\left(v_{1}, v_{2}\right.$, and $\left.v_{3}\right)$ via three mixing angles $\left(\theta_{12}, \theta_{13}\right.$, and $\left.\theta_{23}\right)$. The quantities that govern the oscillation frequencies are two differences between squared masses (i.e., $\delta m^{2}=m_{2}^{2}-m_{1}^{2}>0$ and $\left.\Delta m^{2}=m_{3}^{2}-\left(m_{1}^{2}+m_{2}^{2}\right) / 2\right)$. Central to neutrino studies is understanding the neutrino mass hierarchy (i.e., $\Delta m^{2}>0$ or $\Delta m^{2}<0$ ) (Capozzi et al. 2014; Ge et al. 2013).

Massive (>10 kton) detectors such as the Jiangmen Underground Neutrino Observatory (JUNO) (Li 2014) and Reno-50 (Kim 2013) experiments are being constructed at medium baseline distances (a few tens of kilometers) away from bright reactor antineutrino fluxes in order to assess significant physics goals regarding the neutrino properties, in the first place, the mass hierarchy. These experiments intend also to obtain subpercent precision measurements of neutrino oscillation parameters and along the way make observations of events of astrophysical and terrestrial origin.

JUNO is located (N 22.12, E 112.52 ) in Kaiping, Jiangmen, Guangdong Province (South China), about $53 \mathrm{~km}$ away from the Yangjiang and Taishan nuclear power plants, which are presently under construction. The combined thermal power of these two units is planned to be on the order of 36 GW (Li and Zhou 2014) (Figure 1). The JUNO experiment is designed as a liquid scintillator detector of 20 kton mass that will be built in a laboratory some $700 \mathrm{~m}$ underground (approximately 2,000 $\mathrm{m}$ water equivalent). This amount of overburden will attenuate the cosmic muon flux, which contributes to the overall detector background signal, but this overburden is significantly less than that at the KamLAND and Borexino experiments. The detector energy response and the spatial distribution of the reactor cores are the most critical features affecting the experimental sensitivity (Li et al. 2013) required to achieve the intended physics goals.

The goal of this present study is to predict the geoneutrino signal at JUNO on the basis of an existing reference Earth model (Huang et al. 2013), together with an estimate of the expected reactor antineutrino signal. Since a significant contribution to the expected geoneutrino signal comes from $\mathrm{U}$ and $\mathrm{Th}$ in the continental crust surrounding the site, we follow past approaches to study the local contribution (Coltorti et al. 2011; Fiorentini et al. 2012; Huang et al. 2013; Huang et al. 2014), with a particular interest in focusing on the closest $6^{\circ} \times 4^{\circ}$ grid surrounding the detector. We define this latter region as the local crust (LOC) (Figure 1).

\section{Methods}

The geoneutrino signal expected at JUNO is calculated adopting the same methodology and the same inputs of the reference Earth model developed by Huang et al. (2013). It provides a description of the abundances and distribution of the heat-producing elements (HPEs; i.e., $\mathrm{U}, \mathrm{Th}$, and $\mathrm{K})$ in the Earth's crust, along with their uncertainties. According to this model, the silicate portion of the Earth is composed of five dominant reservoirs: the depleted mantle (DM), the enriched mantle (EM), the lithospheric mantle (LM), the continental crust (CC), and the oceanic crust (OC). The continental crust is dominantly composed of the lower crust (LC), middle crust (MC), and upper crust (UC), and it is overlain by shallow layers of sediments (Sed) which also cover the OC.

The surface geoneutrino flux is calculated by dividing the Earth's surface in $1^{\circ} \times 1^{\circ}$ tiles that are projected vertically into discrete volume cells, and each cell is assigned with physical and chemical states. Just for the sake of computing flux, the $1^{\circ} \times 1^{\circ}$ tiles are further subdivided into many subcells with the same properties of the parent tile. The number of subcells is progressively bigger approaching the detector location with the aim of not introducing any bias due to discretization.

The total crustal thickness of each cell and its associated uncertainty correspond, respectively, to the mean and the half range of three crustal models obtained from different approaches: the global crustal model based on reflection and refraction data 'CRUST 2.0' (Bassin et al. 2000; Laske et al. 2001), the global shear velocity model of the crust and upper mantle 'CUB 2.0' (Shapiro and Ritzwoller 2002), and the high-resolution map of Moho (crust-mantle boundary) depth based on the gravity field data 'GEMMA' (Reguzzoni and Tselfes 2009; Reguzzoni 


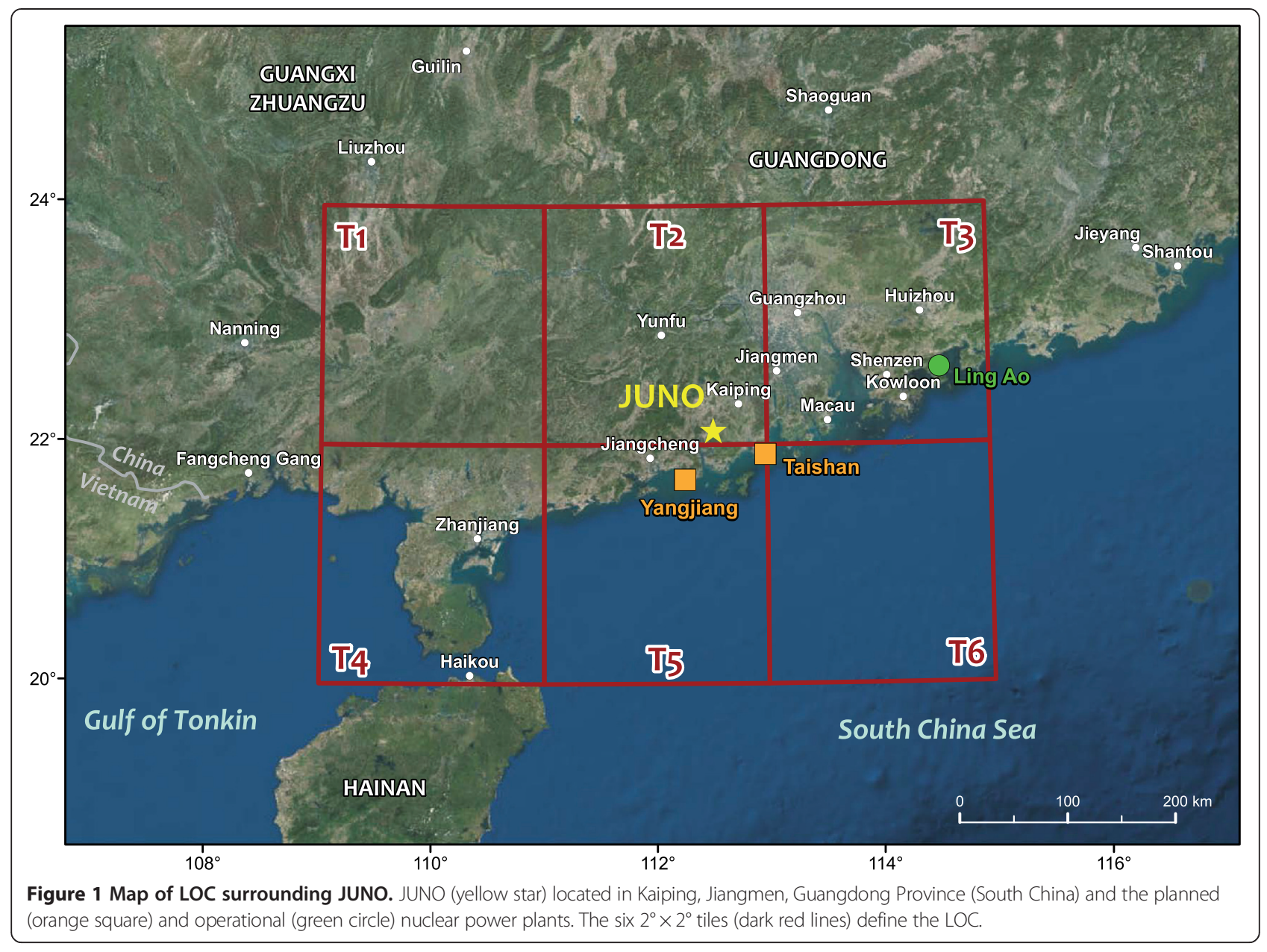

and Sampietro 2015). The reference model incorporates the relative proportional thickness of the crustal layers along with density and elastic properties (compressional and shear wave velocity) reported in CRUST 2.0. The same information is adopted for the Sed layer using the global sediment map of Laske and Masters (1997). In Figure 2, the thicknesses of the continental crust layers in the 24 cells constituting the LOC for JUNO are reported. Their total crustal thickness ranges between 26.3 and $32.3 \mathrm{~km}$ with an uncertainty for each cell of approximately $7 \%$.

The HPE abundances in the Sed, OC, and UC layers are assumed to be relatively homogenous and correspond to the values reported in Table three of Huang et al. (2013). The ratio between the felsic and mafic components in the deep CC (MC and LC) is inferred from seismic velocity data, and these data are in turn used to estimate the $U$ and Th content of each cell of the reference crustal model. Focusing on the LOC, the central values of $U$ abundance in $\mathrm{MC}$ and $\mathrm{LC}$ vary in the range 0.8 to $1.2 \mu \mathrm{g} / \mathrm{g}$ and 0.3 to $0.1 \mu \mathrm{g} / \mathrm{g}$, respectively. The $\mathrm{Th} / \mathrm{U}$ ratio in the deep CC of the LOC is typically approximately 5 as compared to a bulk silicate Earth ratio of 3.9 or a bulk $\mathrm{CC}$ ratio just greater than 4.0; the higher $\mathrm{Th} / \mathrm{U}$ ratio in the deep $\mathrm{CC}$ is likely due to the greater upward mobility of $U$ during dehydration reactions that accompany granulite facies metamorphism of the deep CC.

In the reference model of Huang et al. (2013), the LM corresponds to the portion of the Earth between the Moho discontinuity and an assumed standard depth of $175 \mathrm{~km}$ beneath the surface. The thickness of this unit in the LOC ranges between 143 and $149 \mathrm{~km}$, and its composition is modeled from the database reported in McDonough (1990) and the update in Huang et al. (2013). In our calculation, we adopt for the LM the $\mathrm{U}$ and Th abundances of $0.03_{-0.02}^{+0.05}$ and $0.15_{-0.10}^{+0.28} \mu \mathrm{g} / \mathrm{g}$, respectively (Huang et al. 2013).

The sublithospheric mantle extends down from the base of the lithosphere to the core-mantle boundary and is divided in two spherically symmetric domains, the DM and the EM, whose density profiles are derived from the Preliminary Reference Earth Model (PREM) (Dziewonski and Anderson 1981). Adopting a mass ratio $M_{\mathrm{DM}} / M_{\mathrm{EM}}=4.56$ (Huang et al. 2013), we calculate the 


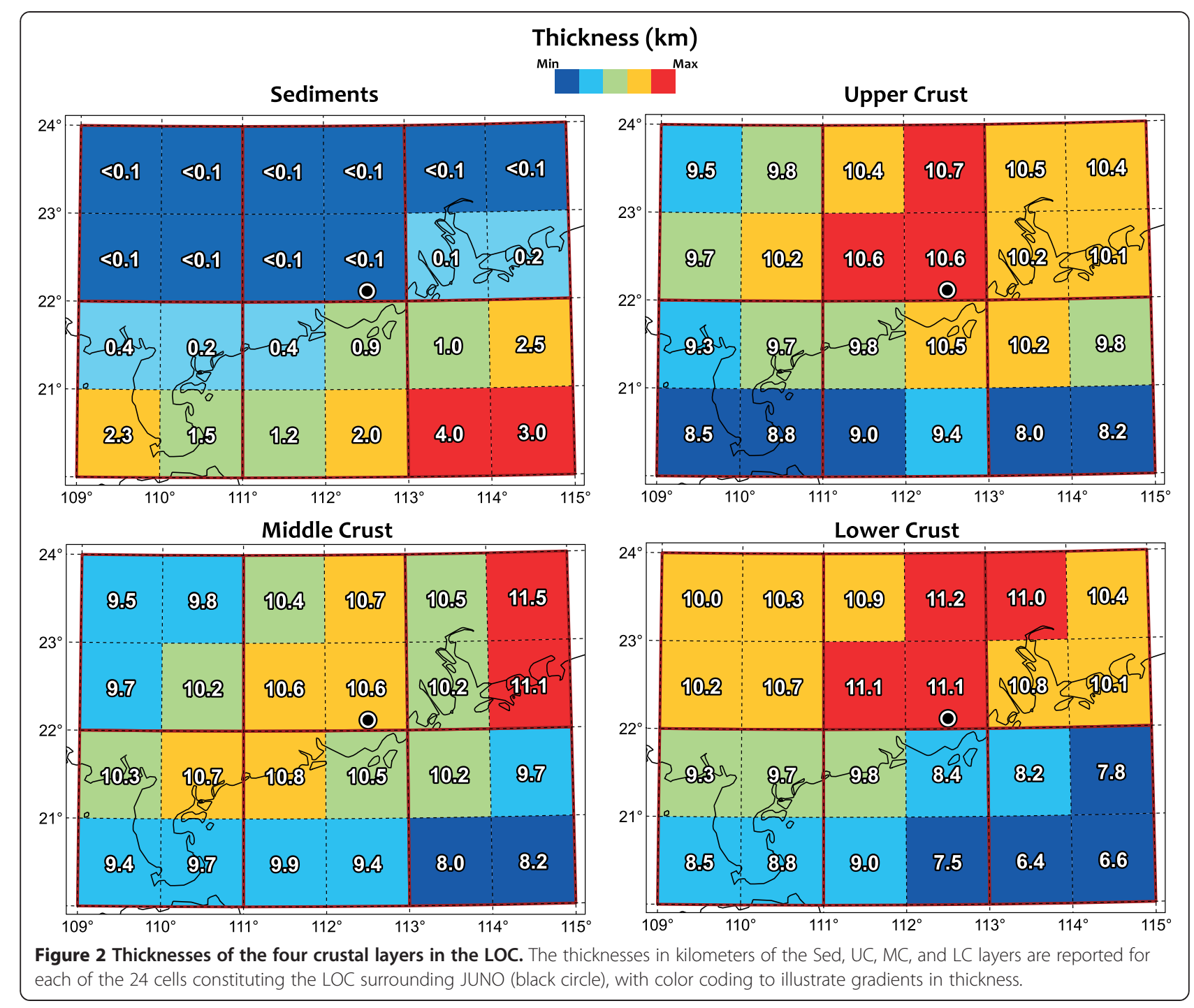

masses of these two reservoirs $M_{\mathrm{DM}}=3.207 \times 10^{24} \mathrm{~kg}$ and $M_{\mathrm{EM}}=0.704 \times 10^{24} \mathrm{~kg}$. In a survey of the midocean ridge basalts (MORB), Arevalo and McDonough (2010) reported the lognormal-based average abundances of uranium $\left(\mathrm{U}_{\mathrm{MORB}}=80 \mathrm{ng} / \mathrm{g}\right)$ and thorium $\left(\mathrm{Th}_{\mathrm{MORB}}=220 \mathrm{ng} / \mathrm{g}\right)$ and, from this, calculated the $\mathrm{U}_{\mathrm{DM}}=8 \mathrm{ng} / \mathrm{g}$ and $\mathrm{Th}_{\mathrm{DM}}=$ $22 \mathrm{ng} / \mathrm{g}$ based on a simple melting model. Based on these assumptions, the $\mathrm{U}_{\mathrm{EM}}$ can be calculated:

$$
\mathrm{U}_{\mathrm{EM}}=\frac{m_{\mathrm{BSE}}-m_{\mathrm{C}}}{M_{\mathrm{EM}}}-\mathrm{U}_{\mathrm{DM}} \frac{M_{\mathrm{DM}}}{M_{\mathrm{EM}}},
$$

where $m_{\mathrm{BSE}}=8.1 \times 10^{16} \mathrm{~kg}$ is the $\mathrm{U}$ mass in the bulk silicate earth (BSE) (McDonough and Sun 1995) and $m_{\mathrm{C}}=3.1 \times 10^{16} \mathrm{~kg}$ is the total $\mathrm{U}$ mass in the crust (Huang et al. 2013). The mantle geoneutrino signals reported in Table 1 are calculated with $U_{D M}=8 \mathrm{ng} / \mathrm{g}$ and $\mathrm{U}_{\mathrm{EM}}=34 \mathrm{ng} / \mathrm{g}$ together with $(\mathrm{Th} / \mathrm{U})_{\mathrm{DM}}=2.8$ and $(\mathrm{Th} / \mathrm{U})_{\mathrm{EM}}=4.8$.
Table 1 Geoneutrino signals from $U$ and Th expected in JUNO

\begin{tabular}{lccc}
\hline & $\boldsymbol{S}(\mathbf{U})$ & $\boldsymbol{S}(\mathbf{T h})$ & $\boldsymbol{S}(\mathbf{U}+\mathbf{T h})$ \\
\hline Sed CC & $0.48_{-0.06}^{+0.06}$ & $0.16_{-0.02}^{+0.02}$ & $0.64_{-0.06}^{+0.06}$ \\
UC & $14.6_{-3.4}^{+3.5}$ & $3.9_{-0.5}^{+0.5}$ & $18.5_{-3.4}^{+3.6}$ \\
MC & $4.7_{-1.8}^{+3.0}$ & $1.7_{-0.8}^{+1.6}$ & $6.8_{-2.3}^{+3.6}$ \\
LC & $0.9_{-0.4}^{+0.7}$ & $0.4_{-0.2}^{+0.7}$ & $1.5_{-0.6}^{+1.0}$ \\
Sed OC & $0.08_{-0.02}^{+0.02}$ & $0.03_{-0.01}^{+0.01}$ & $0.11_{-0.02}^{+0.02}$ \\
OC & $0.05_{-0.02}^{+0.02}$ & $0.01_{-0.01}^{+0.01}$ & $0.06_{-0.02}^{+0.02}$ \\
Bulk crust & $21.3_{-4.2}^{+4.8}$ & $6.6_{-1.2}^{+1.9}$ & $28.2_{-4.5}^{+5.2}$ \\
CLM & $1.3_{-0.9}^{+2.4}$ & $0.4_{-0.3}^{+1.0}$ & $2.1_{-1.3}^{+2.9}$ \\
Total lithosphere & $23.2_{-4.8}^{+5.9}$ & $7.3_{-1.5}^{+2.4}$ & $30.9_{-5.2}^{+6.5}$ \\
DM & 4.2 & 0.8 & 5.0 \\
EM & 2.9 & 0.9 & 3.8 \\
Grand total & $30.3_{-4.8}^{+5.9}$ & $9.0_{-1.5}^{+2.4}$ & $39.7_{-5.2}^{+6.5}$ \\
\hline The
\end{tabular}

The inputs for the calculations are taken from Huang et al. (2013), and the signals from the different reservoirs indicated in the first column are in TNU. 


\section{Results and discussion}

In Table 1, we summarize the contributions to the expected geoneutrino signal at JUNO produced by $\mathrm{U}$ and Th in each of the reservoirs identified in the model. The central value and the asymmetric uncertainties are, respectively, the median and $1 \sigma$ errors of a positively skewed distribution obtained from Monte Carlo simulation. This approach was developed for the first time by Huang et al. (2013) in order to combine the Gaussian probability density function of geophysical and (some) geochemical inputs, together with the lognormal distributions of $U$ and $T h$ abundances observed in the felsic and mafic rocks of $\mathrm{MC}$ and $\mathrm{LC}$.

The total geoneutrino signal at JUNO is $G=39.7_{-5.2}^{+6.5}$ TNU where the $1 \sigma$ error only recognizes the uncertainties of the inputs of the lithosphere, which are mainly due to the uncertainties in the composition of the rocks and subsequently to the geophysical inputs. The predicted mantle contribution at JUNO is assumed to be $S_{\mathrm{M}} \approx 9$ TNU (Huang et al. 2013). The expected geoneutrino signal from the mantle is essentially model dependent, and it is estimated according to a mass balance argument. Uncertainty in the assumed mantle model is much less than the predicted for the lithosphere (e.g., $\delta G \approx \pm 6 \mathrm{TNU}$ ). An extensive discussion of different mantle structures is described in Šrámek et al. (2013), which considers a range of geoneutrino signals for different mantle models.

Thus, a future refinement of the abundances and distribution of HPEs in the UC surrounding the JUNO detector is strongly recommended, as this region provides approximately $47 \%$ of the total geoneutrino signal $(G)$ and is a significant contributor to the total uncertainty.

Plotting the cumulative geoneutrino signal as a function of the distance from JUNO for the different Earth reservoirs (Figure 3), we observe that half of the total signal comes from $\mathrm{U}$ and $\mathrm{Th}$ in the regional crust that lies within $550 \mathrm{~km}$ of the detector. Since the modeling of the geoneutrino flux is based on $1^{\circ} \times 1^{\circ}$ cells, we study the signal produced in LOC subdivided in six $2^{\circ} \times 2^{\circ}$ tiles (Figure 1 ).

The geoneutrino signals from $\mathrm{U}$ and $\mathrm{Th}$ in the lithosphere of each tile are reported in Table 2 with their uncertainties. The main contribution (27\% of $G$ ) comes from tile T2 in which the JUNO experiment is located (Figure 1). The thick UC in this tile, which is covered by a very shallow layer of Sed (Figure 2), is predicted to give a signal of $7.6_{-1.4}^{+1.5}$ TNU. Therefore, a refined study of the $\mathrm{U}$ and $\mathrm{Th}$ content of the $\mathrm{UC}$ in tile T2 is a highvalue target for improving the accuracy and precision of the predicted geoneutrino signal at JUNO. Evaluating the antineutrino signal requires knowledge of several ingredients necessary for modeling the three antineutrino life stages: production, propagation to the detector site,

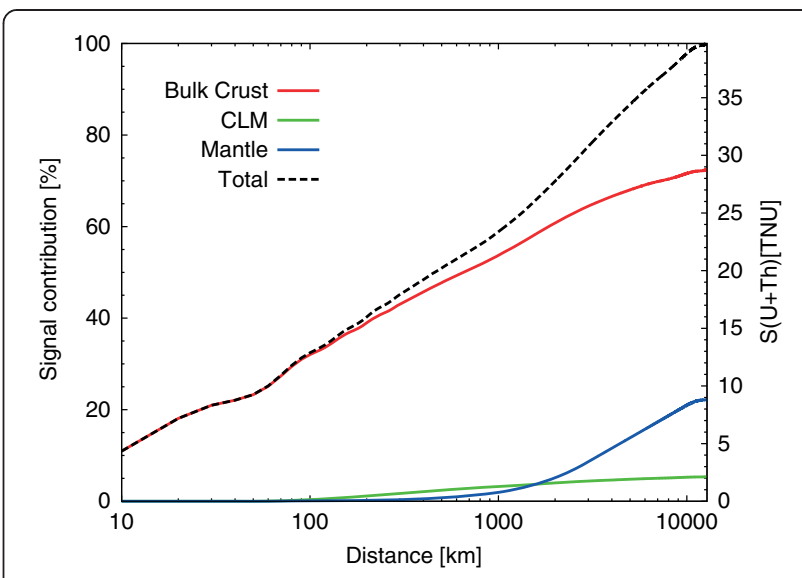

Figure 3 Geoneutrino signal contribution. The cumulative geoneutrino signal and the percentage contributions of the bulk crust, continental lithospheric mantle (CLM), and mantle are represented as functions of the distance from JUNO.

and detection in liquid scintillation detectors via the IBD reaction. The propagation and detection processes are independent from the source of the particles, and we modeled these two stages using the oscillation parameters from Ge et al. (2013) and the IBD cross section from Strumia and Vissani (2003). The spectral parameters for $\mathrm{U}$ and $\mathrm{Th}$ geoneutrinos are from Fiorentini et al. (2007), and the modulation of these fluxes are based on Huang et al. (2013). Reactor antineutrino production is calculated adopting the data from a worldwide reference model from Baldoncini et al. (2015). Reported in Figure 4 are the energy distributions of geoneutrinos and reactor antineutrino signals in two different scenarios: in the full energy region, $R_{\mathrm{OFF}}=95.3_{-2.4}^{+2.6}$ TNU is obtained with data from the worldwide commercial reactors operating in 2013 and $R_{\mathrm{ON}}=1,566_{-100}^{+111} \mathrm{TNU}$, including the Yangjiang (17.4 GW) and Taishan (18.4 GW) nuclear power plants operating at an $80 \%$ annual average load factor (Baldoncini et al. 2015). In the geoneutrino energy window (i.e., 1.806 to $3.272 \mathrm{MeV}$ ), the reactor signals are $S_{\mathrm{OFF}}=26.0_{-2.3}^{+2.2}$ and $S_{\mathrm{ON}}=354_{-41}^{+45}$ TNU (Table 3).

Table 2 Geoneutrino signals from six tiles of the LOC

\begin{tabular}{lcccc}
\hline Tile & $\boldsymbol{S}(\mathbf{U})$ & $\boldsymbol{S}(\mathrm{Th})$ & $\boldsymbol{S}(\mathbf{U}+\mathbf{T h})$ & Percentage \\
\hline T1 & $0.4_{-0.1}^{+0.1}$ & $0.1_{-0.1}^{+0.1}$ & $0.5_{-0.1}^{+0.1}$ & 3.0 \\
T2 & $8.1_{-1.7}^{+1.9}$ & $2.6_{-0.5}^{+0.8}$ & $10.8_{-1.8}^{+2.1}$ & 62.1 \\
T3 & $1.1_{-0.2}^{+0.3}$ & $0.4_{-0.1}^{+0.2}$ & $1.5_{-0.3}^{+0.3}$ & 8.6 \\
T4 & $0.3_{-0.1}^{+0.1}$ & $0.1_{-0.1}^{+0.1}$ & $0.4_{-0.1}^{+0.1}$ & 2.2 \\
T5 & $2.5_{-0.5}^{+0.5}$ & $0.7_{-0.1}^{+0.2}$ & $3.2_{-0.5}^{+0.6}$ & 18.2 \\
T6 & $0.8_{-0.2}^{+0.2}$ & $0.2_{-0.1}^{+0.1}$ & $1.0_{-0.2}^{+0.2}$ & 5.9 \\
\hline
\end{tabular}

The expected geoneutrino signals from $U$ and Th contained in the lithosphere $(C C+C L M)$ of the six tiles reported in Figure 1 are expressed in TNU. In the last column contributions in percentage are reported. 


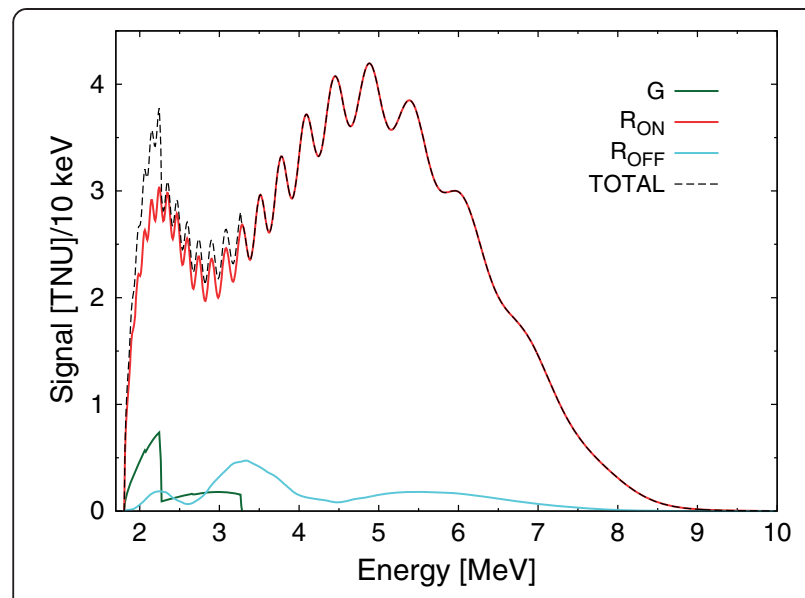

Figure 4 Antineutrino energy spectra expected at JUNO.

Geoneutrino energy spectrum (green) is reported together with the energy reactor antineutrino spectra computed considering the commercial reactors operating all over the world in 2013 (cyan) and adding the contribution of the Yangjiang and Taishan nuclear power plants (red). The reactor antineutrino spectra are computed assuming normal hierarchy and neutrino oscillation. The total spectrum (black dashed lines) is obtained assuming the $R_{\mathrm{ON}}$ scenario.

Assuming a scenario whereby JUNO's signal does not have a background signal from Yangjiang and Taishan nuclear power plants, the ratio of $S_{\mathrm{OFF}} / G=0.7$, which compares to a value of 0.6 for the Borexino detector (Baldoncini et al. 2015). Considering only the statistical uncertainties, in the $R_{\mathrm{OFF}}$ scenario, JUNO is an excellent experiment for geoneutrino measurements reaching a $10 \%$ accuracy on the geoneutrino signal in approximately 105 days (assuming a $\mathrm{C}_{17} \mathrm{H}_{28}$ liquid scintillator composition, a $100 \%$ detection efficiency, and reactor antineutrinos as the sole source of background), given 576 geoneutrino events per year for a target mass of $14.5 \times 10^{32}$ free protons. This optimistic expectation does not take into account the uncertainties of $S_{\mathrm{OFF}}$ and the background due to

Table 3 Geoneutrino and reactor antineutrinos signals at JUNO

\begin{tabular}{lc}
\hline & $\mathbf{S}(\mathrm{TNU})$ \\
\hline Local contribution & $17.4_{-2.8}^{+3.3}$ \\
Far-field crust & $13.4_{-2.4}^{+3.3}$ \\
Mantle & 8.8 \\
Grand total of geoneutrinos & $39.7_{-5.2}^{+6.5}$ \\
Reactors OFF & $26.0_{-2.3}^{+2.2}$ \\
Reactors ON & $354_{-41}^{+45}$ \\
\hline
\end{tabular}

The total geoneutrino signal $(G)$ is the sum of the contributions from the local lithosphere $\left(S_{\mathrm{LOC}}\right)$, from the rest of the lithosphere (i.e., far-field crust, $S_{\mathrm{FFC}}$ ), and from the mantle $\left(S_{M}\right)$. The reactor antineutrino signal in the geoneutrino window is calculated from the data for commercial reactors operating all over the world in $2013\left(S_{\text {OFF }}\right)$ and adding the contribution of the Yangjiang $(17.4 \mathrm{GW})$ and Taishan (18.4 GW) nuclear power plants ( $S_{\mathrm{ON}}$ ) (Baldoncini et al. 2015). All the signals are expressed in TNU. production of cosmic muon spallation, accidental coincidences, and radioactive contaminants in the detector.

\section{Conclusions}

Designed as a 20 kton liquid scintillator detector, the JUNO experiment will collect high statistics for antineutrino signals from reactors and from the Earth. In this study, we focused on predicting the geoneutrino signal using the Earth reference model of Huang et al. (2013). The contribution originating from naturally occurring $U$ and Th in the $6^{\circ} \times 4^{\circ} \mathrm{LOC}$ surrounding the JUNO detector (Figure 1) was determined. The main results of this study are summarized as follows:

- The thicknesses of the Sed, UC, MC, and LC layers of the $241^{\circ} \times 1^{\circ}$ cells of the LOC are reported (Figure 2). The Moho depth of the continental LOC ranges between 26.3 and $32.3 \mathrm{~km}$, and the uncertainty for each $1^{\circ} \times 1^{\circ}$ cell is of the order of $7 \%$.

- The total and local geoneutrino signals at JUNO are $G=39.7_{-5.2}^{+6.5}$ and $S_{\mathrm{LOC}}=17.4_{-2.8}^{+3.3} \mathrm{TNU}$, respectively. The asymmetric $1 \sigma$ errors are obtained from the Monte Carlo simulations and account only for uncertainties from the lithosphere. The major source of uncertainty comes from predicting the abundances and distribution of $\mathrm{U}$ and $\mathrm{Th}$ in local crustal rocks.

- High-resolution seismic data acquired in the LOC can improve the present geophysical model of the crust and CLM, of which the latter is assumed to have a homogenous depth of $175 \pm 75 \mathrm{~km}$. The CLM composition is derived from data for $\mathrm{U}$ and $\mathrm{Th}$ abundances inferred from the peridotite xenoliths, and its geoneutrino signal is of $2.1_{-1.3}^{+2.9} \mathrm{TNU}$.

- The HPEs in the regional crust extending out to $550 \mathrm{~km}$ from the detector produce half of the total expected geoneutrino signal (Figure 3 ). The $\mathrm{U}$ and Th in the $2^{\circ} \times 2^{\circ}$ tile that hosts JUNO produces $10.8_{-1.8}^{+2.1} \mathrm{TNU}$ corresponding to $27 \%$ of $G$. Since this region is characterized by a thick $\mathrm{UC}$, which gives $7.6_{-1.4}^{+1.5} \mathrm{TNU}$, a refined geophysical and geochemical model of the UC of this tile is highly desired.

- The reactor signal in the geoneutrino window assuming two scenarios is $S_{\mathrm{OFF}}=26.0_{-2.3}^{+2.2} \mathrm{TNU}$ with the 2013 reactor operational data only and $S_{\mathrm{ON}}=355_{-41}^{+44} \mathrm{TNU}$ when the contributions of the Yangiiang and Taishan nuclear power plants are added. There is a potential to achieve up to $10 \%$ accuracy on geoneutrinos after 105 days of data accumulation, under conditions of the Yangjiang and Taishan nuclear power plants being off.

The JUNO experiment has the potential to reach a milestone in geoneutrino science, although some technical challenges must be addressed to minimize background 
(e.g., production of cosmic muon spallation, accidental coincidences, and radioactive contaminants in the detector). Assuming $S_{\mathrm{OFF}} / G=0.7$, JUNO can collect hundreds of low-background geoneutrino events in less than a year under optimal conditions. A future refinement of the $U$ and Th distribution and abundance in the LOC is strongly recommended. Such data will lead to insights on the radiogenic heat production in the Earth, the composition of the mantle, and constraints on the chondritic building blocks that made the planet.

\section{Abbreviations}

BSE: bulk silicate Earth; CC: continental crust; CLM: continental lithospheric mantle; DM: depleted mantle; EM: enriched mantle; HPEs: heat-producing elements; IBD: inverse beta decay; JUNO: Jiangmen Underground Neutrino Observatory; LC: lower crust; LM: lithospheric mantle; MC: middle crust; OC: oceanic crust; Sed: sediments; TNU: terrestrial neutrino unit; UC: upper crust.

\section{Competing interests}

The authors declare that they have no competing interests.

\section{Authors' contributions}

VS and FM proposed and conceived the study and produced the geoneutrino data with WFM. MB and BR produced the reactor antineutrino data. IC and GX participated in the data analysis and interpretation of the results. VS took the lead in designing and composing the manuscript, and all the authors contributed to it. All authors read and approved the final manuscript.

\section{Authors' information}

The authors have a geological and physics background. VS and MB are graduate students attending PhD course in Physics at Physics and Earth Sciences Department of the University of Ferrara. FM and BR are researchers of Physics and Earth Sciences Department of the University of Ferrara and INFN. IC and GX are researchers at Legnaro National Laboratories. WFM is a professor of the Department of Geology at the University of Maryland.

\section{Acknowledgements}

We are grateful to R. L. Rudnick and Y. Huang for their fruitful discussions on crustal modeling of geoneutrino fluxes. We appreciate the observations on the geoneutrino signal predictions from S. Dye, G. Fiorentini, L. Ludhova, and $\mathrm{H}$. Watanabe. We thank J. Mandula for the valuable help in compiling the nuclear reactor database. We wish to thank two anonymous reviewers for their detailed and thoughtful reviews. This work was partially supported by the Istituto Nazionale di Fisica Nucleare (INFN) through the ITALRAD Project, by the University of Ferrara through the research initiative 'Fondo di Ateneo per la Ricerca scientifica FAR 2014' and partially by the U.S. National Science Foundation Grants EAR 1067983/1068097.

\section{Author details}

1 Department of Physics and Earth Sciences, University of Ferrara, Via Saragat 1, 44121 Ferrara, Italy. ${ }^{2}$ Legnaro National Laboratories, INFN, Viale dell'Università, 2, 35020 Legnaro, Italy. ${ }^{3}$ Ferrara Section, INFN, Via Saragat 1, 44121 Ferrara, Italy. ${ }^{4}$ Department of Geology, University of Maryland, 237 Regents Drive, College Park, MD 20742, USA.

Received: 5 December 2014 Accepted: 1 March 2015

\section{Published online: 18 March 2015}

\section{References}

Arevalo R, McDonough WF (2010) Chemical variations and regional diversity observed in MORB. Chem Geol 271(1-2):70-85. doi:10.1016/j.chemgeo.2009.12.013

Baldoncini M, Callegari I, Fiorentini G, Mantovani F, Ricci B, Strati V, Xhixha G (2015) Reference worldwide model for antineutrinos from reactors. Physical Review D 91(6):065002

Bassin C, Laske G, Masters TG (2000) The current limits of resolution for surface wave tomography in North America. EOS Trans AGU 81:F897

Borexino Collaboration (2013) Measurement of geo-neutrinos from 1353 days of Borexino. Phys Lett B 722(4-5):295-300. doi:10.1016/j.physletb.2013.04.030
Capozzi F, Lisi E, Marrone A (2014) Neutrino mass hierarchy and electron neutrino oscillation parameters with one hundred thousand reactor events. Phys Rev D 89(1):013001

Coltorti M, Boraso R, Mantovani F, Morsilli M, Fiorentini G, Riva A, Rusciadelli G, Tassinari R, Tomei C, Di Carlo G, Chubakov V (2011) U and Th content in the Central Apennines continental crust: a contribution to the determination of the geo-neutrinos flux at LNGS. Geochim Cosmochim Acta 75(9):2271-2294. doi:10.1016/j.gca.2011.01.024

Dziewonski AM, Anderson DL (1981) Preliminary reference Earth model. Phys Earth Planet In 25:297-356. doi:10.1016/0031-9201(81)90046-7

Fiorentini G, Fogli G, Lisi E, Mantovani F, Rotunno A (2012) Mantle geoneutrinos in KamLAND and Borexino. Physical Review D 86 (3). doi:10.1103/ PhysRevD.86.033004

Fiorentini G, lanni A, Korga G, Lissia M, Mantovani F, Miramonti L, Oberauer L, Obolensky M, Smirnov O, Suvorov Y (2010) Nuclear physics for geo-neutrino studies. Physical Review C 81(3). doi:10.1103/PhysRevC.81.034602

Fiorentini G, Lissia M, Mantovani F (2007) Geo-neutrinos and Earth's interior. Phys Rep 453(5-6):117-172. doi:10.1016/j.physrep.2007.09.001

Ge S-F, Hagiwara K, Okamura N, Takaesu Y (2013) Determination of mass hierarchy with medium baseline reactor neutrino experiments. J High Energ Phys 2013(5):1-23. doi:10.1007/JHEP05(2013)131

Huang Y, Chubakov V, Mantovani F, Rudnick RL, McDonough WF (2013) A reference Earth model for the heat-producing elements and associated geoneutrino flux. Geochem Geophys Geosyst 14(6):2023-2029. doi:10.1002/ggge.20129

Huang Y, Strati V, Mantovani F, Shirey SB, McDonough WF (2014) Regional study of the Archean to Proterozoic crust at the Sudbury Neutrino Observatory (SNO+), Ontario: predicting the geoneutrino flux. Geochem Geophys Geosyst 15(10):3925-3944. doi:10.1002/2014gc005397

KamLAND Collaboration (2005) Measurement of neutrino oscillation with KamLAND: evidence of spectral distortion. Phys Rev Lett 94(8):08180

KamLAND Collaboration (2013) Reactor on-off antineutrino measurement with KamLAND. Phys Rev D 88(3):033001

Kim S-B (2013) Proposal for RENO-50. In: International workshop on RENO-50, Seoul National University, Korea, 13-14 June 2013

Laske G, Masters TG (1997) A global digital map of sediment thickness. Eos Trans AGU 78:F483

Laske G, Masters TG, Reif C (2001) CRUST 2.0: a new global crustal model at $2 \times 2$ degrees. http://igppweb.ucsd.edu/ gabi/crust2.html

Li Y-F, Cao J, Wang Y, Zhan L (2013) Unambiguous determination of the neutrino mass hierarchy using reactor neutrinos. Phys Rev D 88(1):013008

Li Y-F, Zhou Y-L (2014) Shifts of neutrino oscillation parameters in reactor antineutrino experiments with non-standard interactions. Nucl Phys B 888(0):137-153. doi:10.1016/j.nuclphysb.2014.09.013

Li YF (2014) Overview of the Jiangmen Underground Neutrino Observatory (JUNO). arXiv:1402.6143 [physics.ins-det] http://arxiv.org/pdf/1402.6143

McDonough WF (1990) Constraints on the composition of the continental lithospheric mantle. Earth Planet Sci Lett 101(1):1-18. doi:10.1016/0012-821x (90)90119-i

McDonough WF, Sun S-S (1995) The composition of the Earth. Chem Geol 120:223-253. doi:10.1016/0009-2541(94)00140-4

Reguzzoni M, Sampietro D (2015) GEMMA: an earth crustal model based on GOCE satellite data. Int J Appl Earth Observation Geoinform 35(Part A (0)):31-43. doi:10.1016/j.jag.2014.04.002

Reguzzoni M, Tselfes N (2009) Optimal multi-step collocation: application to the space-wise approach for GOCE data analysis. J Geodes 83(1):13-29. doi:10.1007/s00190-008-0225-x

Shapiro NM, Ritzwoller MH (2002) Monte-Carlo inversion for a global shear-velocity model of the crust and upper mantle. Geophys J Int 151:88-105. doi:10.1046/ j.1365-246X.2002.01742.X

Šrámek O, McDonough WF, Kite ES, Lekić V, Dye ST, Zhong S (2013) Geophysical and geochemical constraints on geoneutrino fluxes from Earth's mantle. Earth Planet Sci Lett 361:356-366. doi:10.1016/j.epsl.2012.11.001

Strumia A, Vissani F (2003) Precise quasielastic neutrino/nucleon cross-section. Phys Lett B 564(1-2):42-54. doi:10.1016/s0370-2693(03)00616-6 